Nuclear Forensics and Attribution for Improved Energy Security: The Use of Taggants in Nuclear Fuel

M. J. Kristo, M. Robel, I. D. Hutcheon

April 12, 2007 
This document was prepared as an account of work sponsored by an agency of the United States Government. Neither the United States Government nor the University of California nor any of their employees, makes any warranty, express or implied, or assumes any legal liability or responsibility for the accuracy, completeness, or usefulness of any information, apparatus, product, or process disclosed, or represents that its use would not infringe privately owned rights. Reference herein to any specific commercial product, process, or service by trade name, trademark, manufacturer, or otherwise, does not necessarily constitute or imply its endorsement, recommendation, or favoring by the United States Government or the University of California. The views and opinions of authors expressed herein do not necessarily state or reflect those of the United States Government or the University of California, and shall not be used for advertising or product endorsement purposes.

This work was performed under the auspices of the U.S. Department of Energy by University of California, Lawrence Livermore National Laboratory under Contract W-7405-Eng-48. 
September 21, 2006

\title{
Nuclear Forensics and Attribution for Improved Energy Security The Use of Taggants in Nuclear Fuel
}

\author{
Michael Kristo, Martin Robel, Ian Hutcheon
}

\section{Introduction}

The Global Nuclear Energy Partnership (GNEP), recently announced by DOE Secretary Bodman, poses significant new challenges with regard to securing, safeguarding, monitoring and tracking nuclear materials. In order to reduce the risk of nuclear proliferation, new technologies must be developed to reduce the risk that nuclear material can be diverted from its intended use. Regardless of the specific nature of the fuel cycle, nuclear forensics and attribution will play key roles to ensure the effectiveness of nonproliferation controls and to deter the likelihood of illicit activities. As the leader of the DHS nuclear and radiological pre-detonation attribution program, LLNL is uniquely positioned to play a national leadership role in this effort.

Ensuring that individuals or organizations engaged in illicit trafficking are rapidly identified and apprehended following theft or diversion of nuclear material provides a strong deterrent against unlawful activities. Key to establishing this deterrent is developing the ability to rapidly and accurately determine the identity, source and prior use history of any interdicted nuclear material.

Taggants offer one potentially effective means for positively identifying lost or stolen nuclear fuels. Taggants are materials that can be encoded with a unique signature and introduced into nuclear fuel during fuel fabrication. During a nuclear forensics investigation, the taggant signature can be recovered and the nuclear material identified through comparison with information stored in an appropriate database. Unlike serial numbers or barcodes, microtaggants can provide positive identification with only partial recovery, providing extreme resistance to any attempt to delete or alter them.

\section{Research Efforts}

In order for a taggant to be effective, the signature must survive long-term irradiation in a nuclear reactor. The signature must, therefore, either be impervious to neutron irradiation or evolve during irradiation in a known way. Consequently, the use of molecular signatures as fuel taggants would be problematic, since molecular species are often degraded or destroyed during irradiation. Elements, and their constituent isotopes, would be more suitable as taggants, because their transmutation under neutron irradiation can be accurately modeled with modern depletion codes.

We have investigated the characteristics of a number of elements for use as potential taggants. We modeled their behavior under neutron irradiation in a 17x17 array pressurized water reactor (PWR) using the Origen-ARP depletion code. ${ }^{1}$ We simulated the irradiation of fuel that contained small amounts of the candidate element (10-250

\footnotetext{
${ }^{1}$ Origen-ARP 2.00, Oak Ridge National Laboratory, CCC-702, available through the Radiation Safety Information Computer Center.
} 
ppmw), in addition to the low-enriched $\left(3 \%{ }^{235} \mathrm{U}\right) \mathrm{UO}_{2}$ "meat." We simulated the irradiation of the fuel at $1 \mathrm{GW}_{\mathrm{e}}\left(\sim 3.3 \mathrm{GW}_{\mathrm{th}}\right)$ for 1 year. We then used a specialized $\mathrm{MATLAB}^{2}$ routine to extract data on the nuclides of interest from the ORIGEN-ARP output file and write that data to an $\mathrm{EXCEL}^{3}$ spreadsheet. For example, we did not explicitly provide for a cooling time in the simulation, but did eliminate nuclides with half-lives shorter than 2 months.

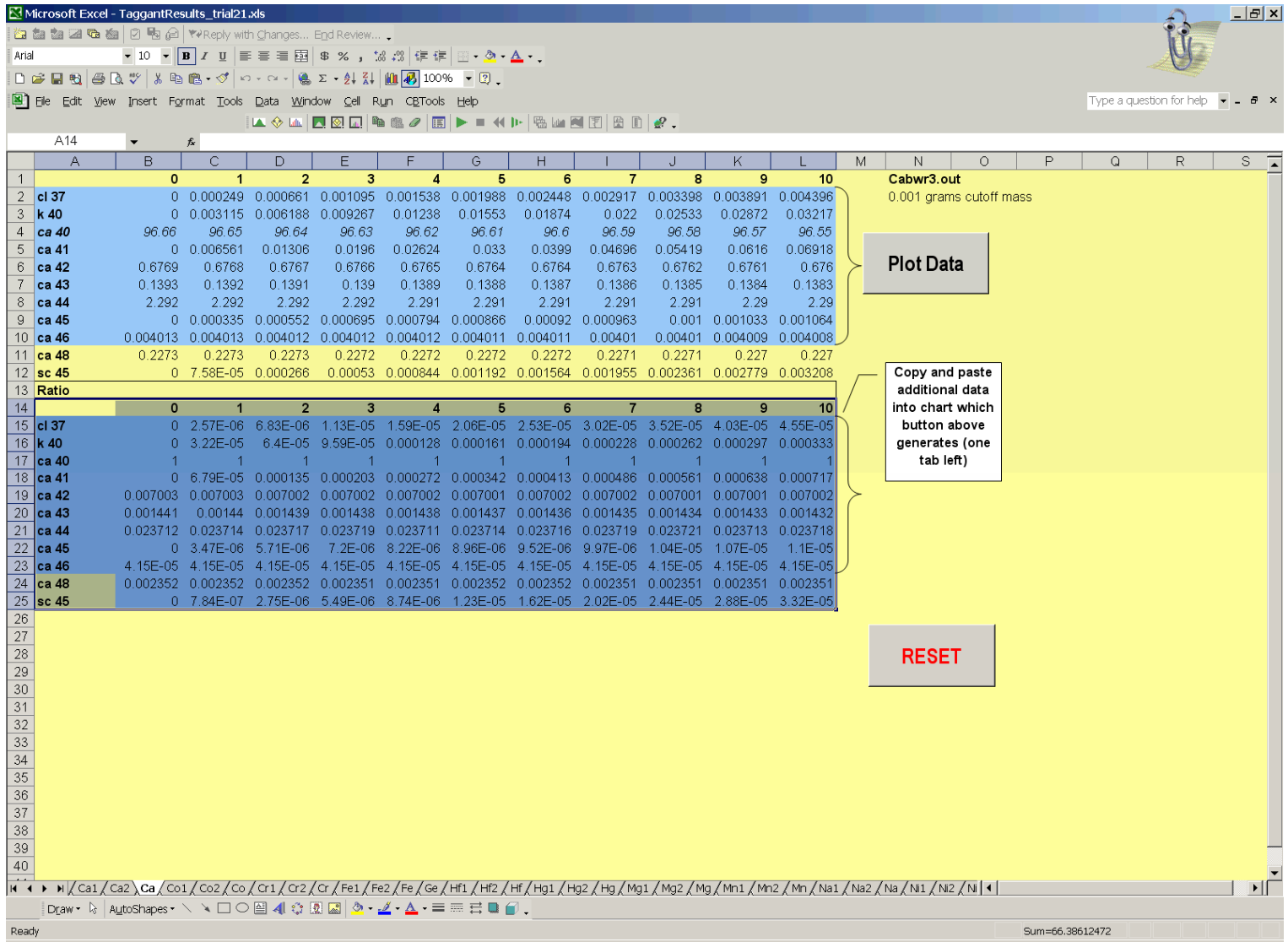

Figure 1.

Excel spreadsheet with data extracted from modeling of $\mathrm{Ca}$ irradiation.

We concentrated our efforts on isotopes that lie on either side of the fission yield curve masses below $\sim 70$ or above $\sim 170$ (see Table 1). By doing so, we sought to avoid the distortion of our taggant signature by isotopes produced from the fission of the $U$ atoms. Even isotopes with relatively low fission yield might easily overwhelm the taggant signature, since the uranium atoms will be present in significant excess $\left(\sim 10^{4}-10^{5}\right.$ times greater).

\footnotetext{
${ }^{2}$ MATLAB R2006b, Mathworks, Natick MA.

${ }^{3}$ EXCEL 2003, Microsoft Corporation, Redmond, WA.
} 
Table 1.

Candidate Elements

\begin{tabular}{|l|l|}
\hline $\mathrm{Ca}$ & $\mathrm{Ni}$ \\
\hline $\mathrm{Co}$ & $\mathrm{Os}$ \\
\hline $\mathrm{Cr}$ & $\mathrm{Pb}$ \\
\hline $\mathrm{Fe}$ & $\mathrm{Pt}$ \\
\hline $\mathrm{Ge}$ & $\mathrm{Si}$ \\
\hline $\mathrm{Hf}$ & $\mathrm{Ti}$ \\
\hline $\mathrm{Hg}$ & $\mathrm{V}$ \\
\hline $\mathrm{Mg}$ & $\mathrm{W}$ \\
\hline $\mathrm{Mn}$ & $\mathrm{Yb}$ \\
\hline $\mathrm{Na}$ & $\mathrm{Zn}$ \\
\hline
\end{tabular}

We evaluated each candidate element as to how each met several desirable characteristics. The most important characteristic was the existence of multiple, naturally occurring isotopes with low neutron absorption cross-sections. The ratios of these isotopes will not change very much during irradiation and can be used to encode information about the fuel. The greater the number of naturally occurring isotopes with low neutron absorption cross-sections, the more information that can be encoded into the signature, just as numbers with more digits can encode more information than numbers with fewer digits. The more information that can be encoded in the taggant, the more precisely the interdicted fuel can be attributed. One might potentially encode information that identified the manufacturer of the fuel, the target customer for the fuel, and even the lot number of the fuel.

Using this same analogy to numbers, the information conveyed by a single isotopic ratio (the digit) is determined by our ability to discriminate between two adjacent values of the initial (unirradiated) isotope ratio. This, in turn, will be determined by the analytical precision of measuring the present (irradiated) isotopic ratio, but, more importantly, the evolution of the ratio during irradiation and our ability to correct for this evolution.

Therefore, a second desirable characteristic would be the existence of a few isotopes of a single element that do change markedly under irradiation. In this case, the relative abundance of these isotopes in the irradiated fuel would serve as a measure of the neutron fluence experienced by the fuel. It is important to have two or more isotopes of the same element, because then the signature can be determined from a relative measurement (the isotopic ratio), rather than an absolute measurement (exact isotopic concentration).

Knowledge of the neutron fluence experienced by the fuel could be then be used to calculate the starting isotopic compositions (signatures) for all of the other species, thus allowing greater precision in the encoding of the signature. The neutron energy spectrum 
is also an important parameter in determining the evolution of the nuclides under irradiation, since it determines the exact cross-section for neutron absorption. However, information on reactor type could be part of the information encoded into the taggant and, therefore, the neutron energy spectrum could be known to a significant extent, especially for commercially available reactors.

Some of these elements will, of course, also be present in the fuel as impurities with natural isotopic abundances. Therefore, a third desirable characteristic is low natural abundance in current nuclear fuel. We used data from our DHS-sponsored U.S. commercial nuclear fuels database to evaluate the level and variability of candidate elements in current fuel pellets. We also noted the upper limit for each element based upon the "Standard Specification for Sintered Uranium Dioxide Pellets" (C776-00). ${ }^{4}$ It is common for elements with little or no abundance in uranium dioxide fuel pellets to have no specified upper limit, since these elements are of no practical concern to the fuel fabrication industry.

The difference between the background level of each element and the upper limit define what we call "headroom." With sufficient headroom, we can be sure that we can add an amount of taggant well in excess of the background level, avoiding excessive distortion of the taggant signature from background impurities, but without causing the fuel to fall outside specifications.

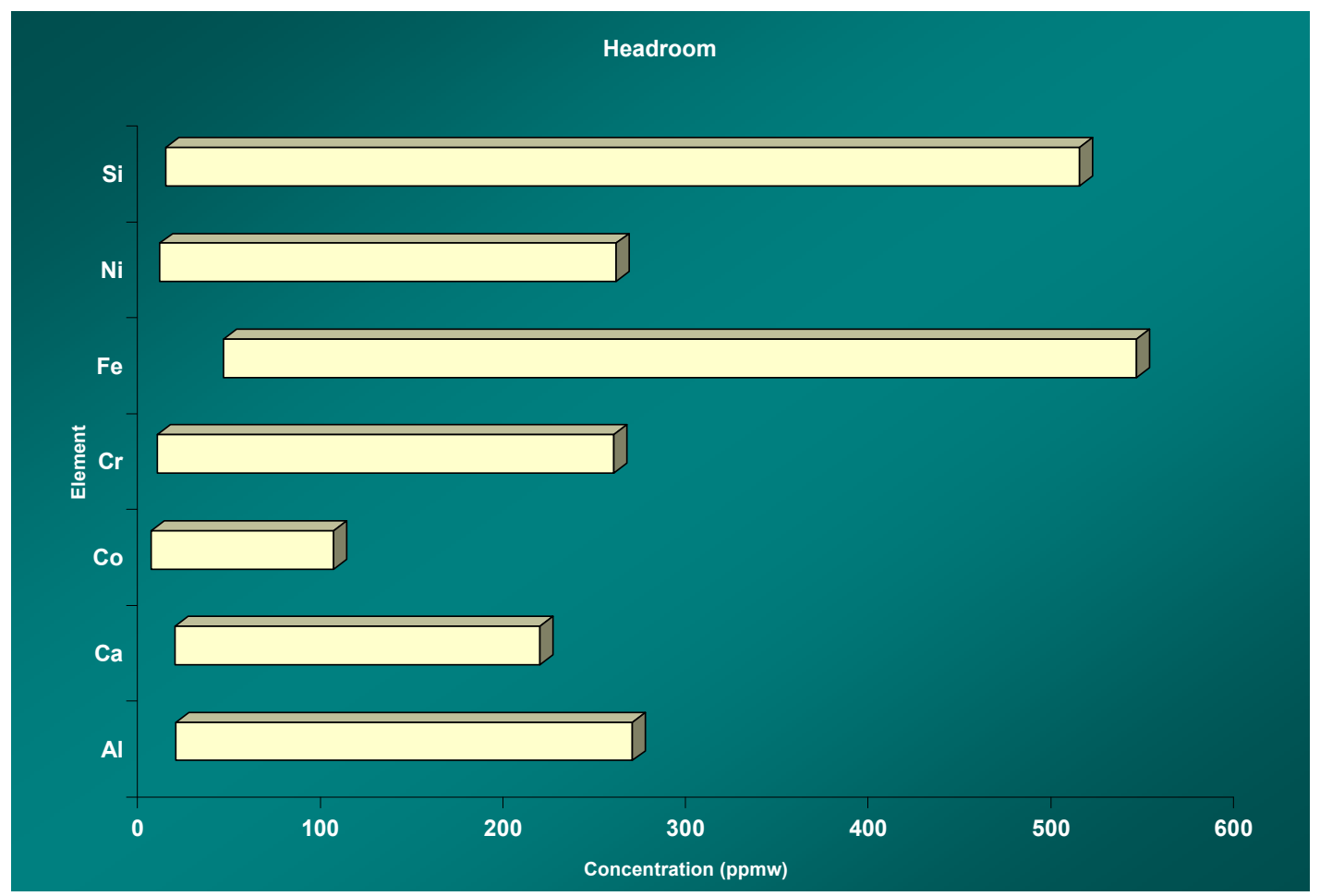

Figure 2.

Graphic representation of headroom for selected elements in $\mathrm{UO}_{2}$ pellets.

\footnotetext{
${ }^{4}$ Annual Book of ASTM Standards, Vol. 12.01, Nuclear Energy, American Society for Testing \& Materials (West Goshohocken, PA: 2003).
} 
A fourth desirable characteristic is the existence of an isotope whose concentration, due to the half-life of the isotope, reaches a plateau during irradiation. After irradiation, the decay of such an isotope can serve as a chronometer for the time since irradiation. This information can be used, along with the other information provided by the taggant, to establish a timeline for the interdicted fuel.

Another key characteristic of a good taggant is the ease of analysis. Since the signature will be isotopic in nature and include both stable and radioactive isotopes, mass spectrometry will be the most likely analytical technique used. Inductively coupled plasma/mass spectrometry (ICP/MS) has both the sensitivity and precision to measure the resulting isotopic abundances. However, we must make sure that the elements of interest can be chemically separated from the bulk material in such a way that the isotopic signatures can be measured without isobaric interferences.

\section{$\underline{\text { Results }}$}

From our initial studies, Ni, Pt, and Os appear to be promising taggants. Figure 3 shows the evolution of the $\mathrm{Ni}$ isotopes and their transmutation products with irradiation time.

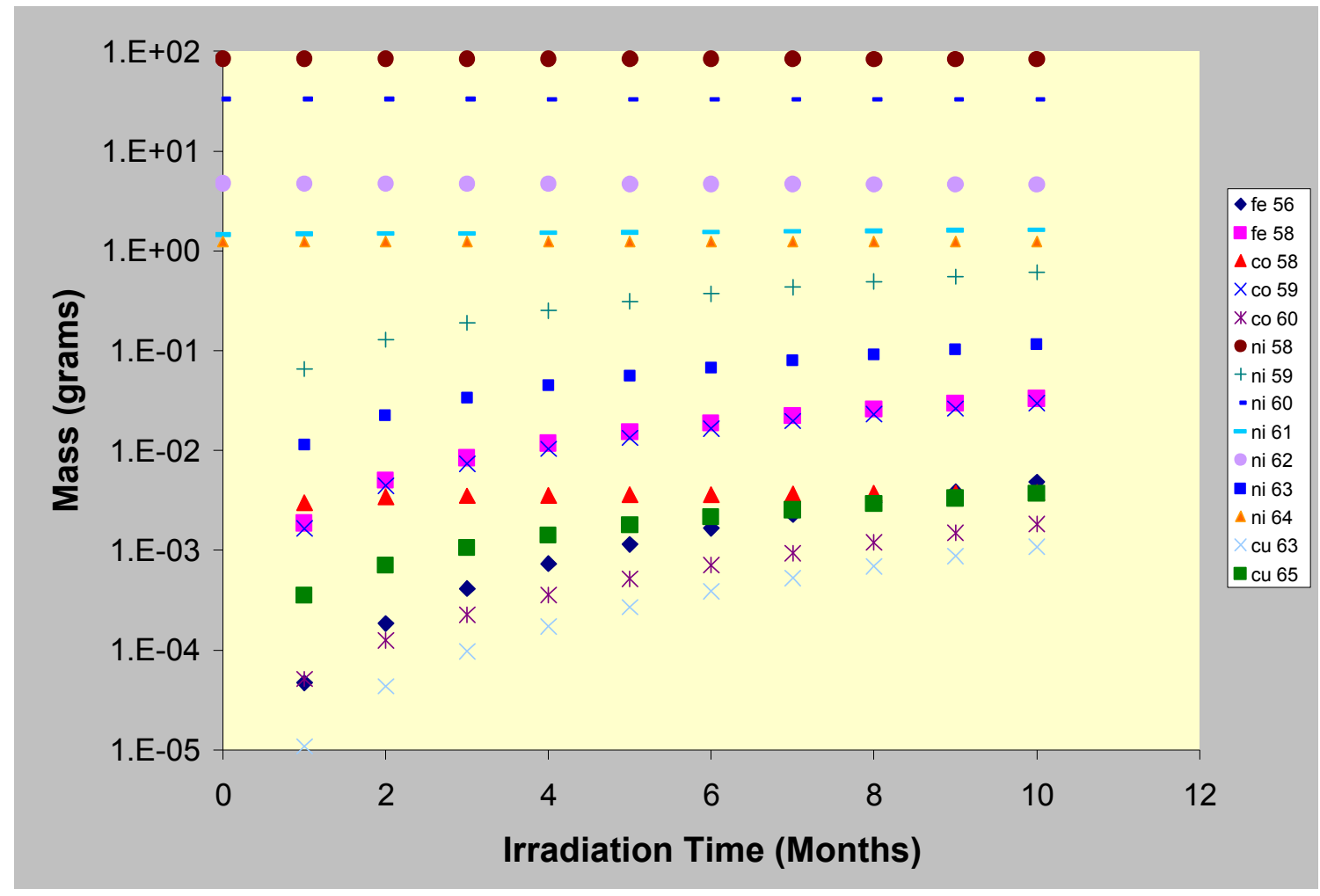

Figure 3.

Irradiation of Ni Taggant in Fuel

Ni has 5 naturally occurring isotopes of low neutron cross-section $\left({ }^{58} \mathrm{Ni},{ }^{60} \mathrm{Ni},{ }^{61} \mathrm{Ni},{ }^{62} \mathrm{Ni}\right.$, ${ }^{64} \mathrm{Ni}$ ), allowing the use of 4 isotopic ratios for encoding information. The ${ }^{63} \mathrm{Cu} /{ }^{65} \mathrm{Cu}$ ratio varies with irradiation time, allowing the determination of neutron fluence in this reactor. The levels of ${ }^{59} \mathrm{Ni}\left(\mathrm{t}_{1 / 2}-7.6 \times 10^{4}\right.$ years $),{ }^{58} \mathrm{Co}\left(\mathrm{t}_{1 / 2}-70.8\right.$ days $)$, and ${ }^{63} \mathrm{Ni}\left(\mathrm{t}_{1 / 2}-101\right.$ years) reach a plateau during the irradiation history. Therefore, after irradiation, the decay of these isotopes can provide a chronometer for the time since irradiation. The differences 
in half-lives between these nuclides allows the determination of the time since irradiation over a large range of times (shorter half-life system for short times; longer half-life system for long times).

Figure 4 shows the evolution of the Pt isotopes and their transmutation products with irradiation time.

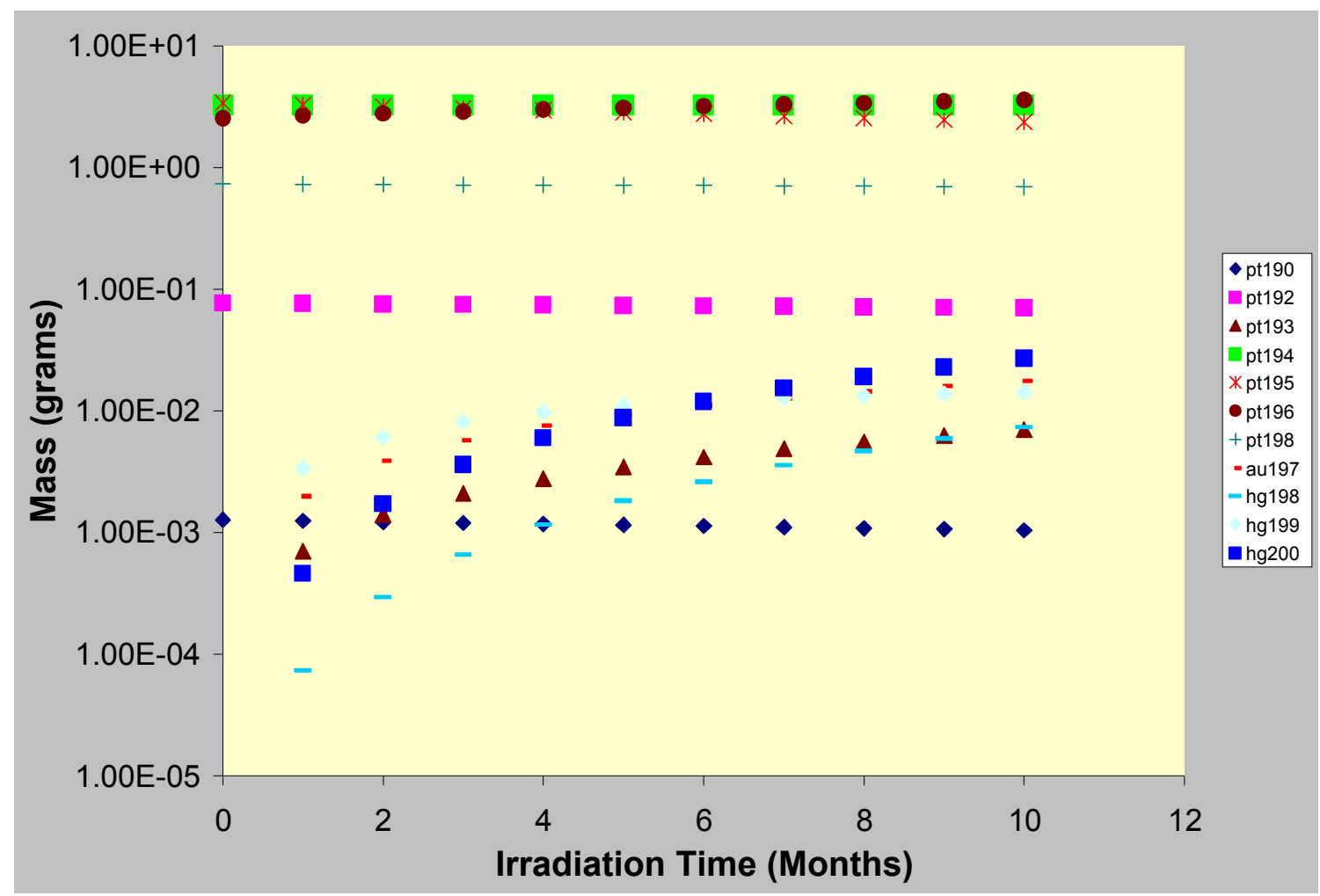

Figure 4.

Irradiation of Pt Taggant in Fuel

Pt has 4 naturally occurring isotopes of low neutron cross-section $\left({ }^{190} \mathrm{Pt},{ }^{192} \mathrm{Pt},{ }^{194} \mathrm{Pt}\right.$, ${ }^{198} \mathrm{Pt}$ ), allowing the use of 3 isotopic ratios for encoding information. This system has 2 sets of isotopes that vary with irradiation time: $\left({ }^{193} \mathrm{Pt},{ }^{195} \mathrm{Pt}\right.$ and $\left.{ }^{196} \mathrm{Pt}\right)$ and $\left({ }^{198} \mathrm{Hg},{ }^{199} \mathrm{Hg}\right.$, and ${ }^{200} \mathrm{Hg}$ ), either of which allow the determination of neutron fluence in the reactor. The levels of ${ }^{193} \mathrm{Pt}\left(\mathrm{t}_{1 / 2}-50\right.$ years) reaches a plateau during the irradiation history. Therefore, after irradiation, the decay of this isotope could provide a chronometer for the time since irradiation.

Figure 5 shows the evolution of the Os isotopes and their transmutation products with irradiation time. 


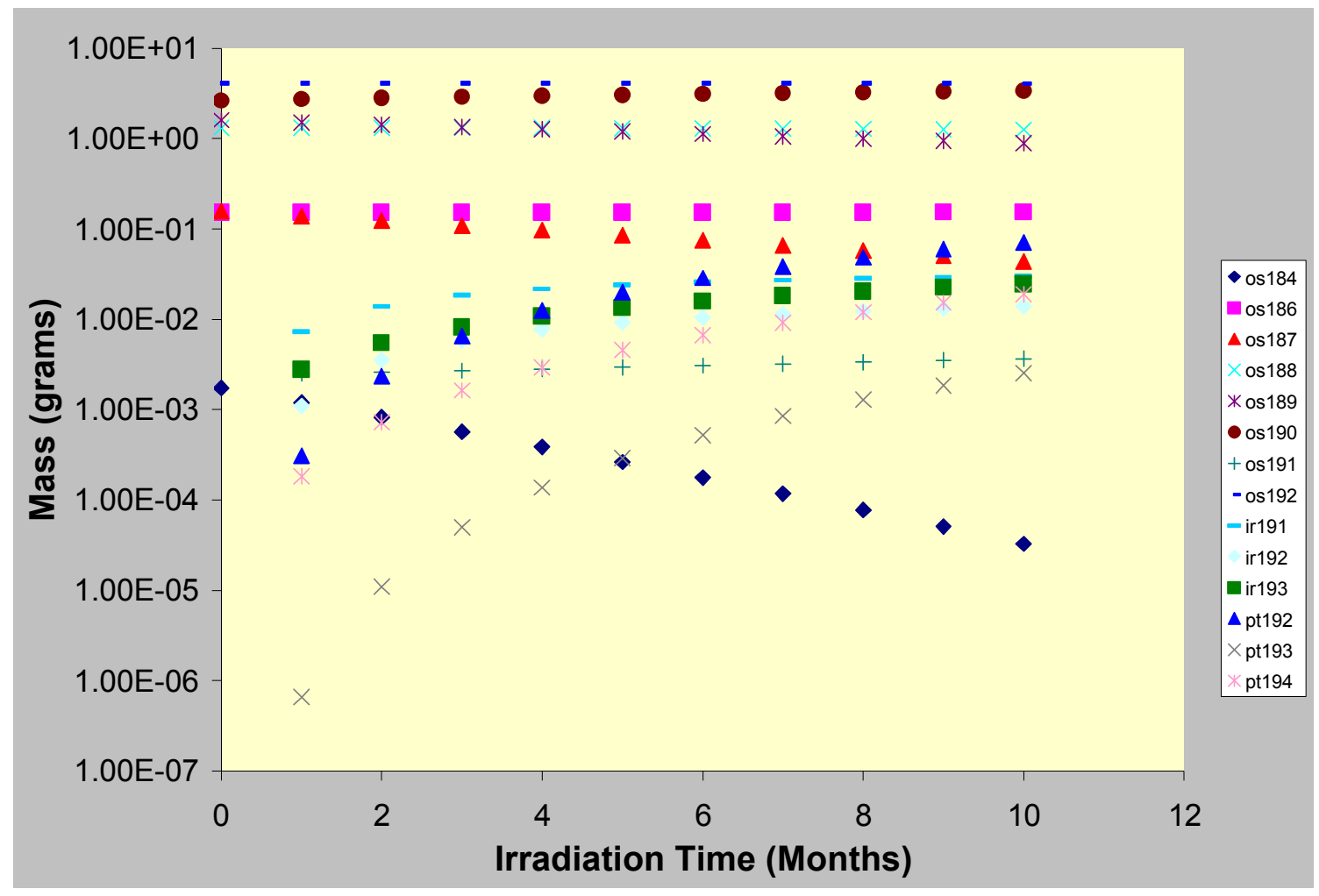

Figure 5 .

Irradiation of Os Taggant in Fuel

Os has 2 naturally occurring isotopes of low neutron cross-section $\left({ }^{186} \mathrm{Os},{ }^{188} \mathrm{Os}\right)$, which allows only the use of a single isotopic ratio for encoding information. However, this system has 2 sets of isotopes that vary with irradiation time: $\left({ }^{184} \mathrm{Os},{ }^{187} \mathrm{Os},{ }^{189} \mathrm{Os},{ }^{190} \mathrm{Os}\right.$, and $\left.{ }^{192} \mathrm{Os}\right)$ and $\left({ }^{191} \mathrm{Ir}\right.$ and $\left.{ }^{193} \mathrm{Ir}\right)$, either of which allow the determination of neutron fluence in the reactor. The levels of ${ }^{192} \operatorname{Ir}\left(\mathrm{t}_{1 / 2}-74\right.$ days $)$ and, to a lesser extent, ${ }^{193} \mathrm{Pt}\left(\mathrm{t}_{1 / 2}-50\right.$ years $)$ reach a plateau during the irradiation history. Therefore, after irradiation, the decay of these isotopes could provide chronometers for the time since irradiation. Again, the differences in half-lives between these nuclides allows the determination of the time since irradiation over a large span of time (shorter half-life system for short times; longer halflife system for long times).

$\mathrm{Ca}, \mathrm{W}$, and Hf are also worth considering, although they lack one or more of our ideal characteristics. The use of $\mathrm{Ca}$ offers 4 isotopic ratios of naturally occurring isotopes of low neutron absorption cross-section. It also has a radioactive isotope whose concentration reaches a plateau during irradiation. However, it does not have a good isotopic system for measuring neutron fluence. The use of Hf or $\mathrm{W}$ offers multiple isotopic systems for measuring neutron fluence, as well isotopes that reach a plateau during irradiation. However, all of the isotope ratios of naturally occurring isotopes vary considerable during irradiation. 


\section{Future Work}

Many of the interdicted samples that we receive at LLNL are reprocessed nuclear fuels. In our research on taggants, we have yet to address how the potential taggants would behave during fuel reprocessing. It is necessary to consider what behavior is, in fact, desirable. Should the taggant follow the U process stream, the Pu stream, or the fission products? We could conceivably add multiple taggants, so that a taggant will follow each of the process streams, but this would clearly add complexity to the fuel design and manufacture. On the one hand, it would be desirable for the taggant to follow $\mathrm{U}$ or $\mathrm{Pu}$, so that the taggant system could avoid being fooled through the use of clandestine reprocessing. On the other hand, if reactor fuel is constantly being reprocessed and mixed, we might have the potential for mixed taggant signatures at some point that might interfere with one another. Both the operations and the chemistry of taggants in nuclear fuels are areas ripe for future research.

We must also address the commercial needs of nuclear fuel manufacturers. In particular, fuel manufacturers will be concerned about the following:

1. Whether the taggant will degrade fuel performance

2. Whether the taggant will create any new safety issues or radiation hazards

3. Whether the taggant will adversely affect manufacturing processes and/or increase costs

4. Whether the taggant will interact with the additives in the fuel in a negative way

The nuclear fuel industry is unlikely to undertake these activities on their own initiative. However, it may be possible, with sufficient research into the materials science of nuclear fuels, to develop a material that both serves as a taggant and actually improves the performance of the fuel itself. This would provide a strong business incentive for manufacturers to incorporate taggants into their commercial nuclear fuels.

Through our DHS nuclear forensics program, we have developed strong working relationships with all three U.S. manufacturers of commercial nuclear fuel. We have identified the key individuals at the fuel fabrication facilities and have developed nondisclosure agreements with all three. The U.S. manufacturers have exhibited a keen awareness of the national need for a robust nuclear forensics program and we have every reason to expect their full cooperation in such a study. 